\title{
(6) OPEN ACCESS \\ Systematic review of $\beta$ blocker, aspirin, and statin in critically ill patients: importance of severity of illness and cardiac troponin
}

\author{
Florence G Rothenberg, ${ }^{1,2}$ Michael B Clay, ${ }^{2}$ Hina Jamali, ${ }^{1}$ \\ Robin H Vandivier-Pletsch ${ }^{1,2}$
}

\begin{abstract}
- Additional material is published online only. To view please visit the journal online (http://dx.doi.org/10. 1136/jim-2016-000374).

${ }^{1}$ University of Cincinnati, Cincinnati, Ohio, USA ${ }^{2}$ Cincinnati Veterans Administration Medical Center, Cincinnati, Ohio, USA
\end{abstract}

\section{Correspondence to} Florence G Rothenberg, University of Cincinnati, 231 Albert Sabin Way, ML 0542, Cincinnati, OH 45267, USA; florence.rothenberg@uc.edu

Accepted 2 January 2017 Published Online First 30 January 2017

Copyright (C) 2016 American Federation for Medical Research

\section{ABSTRACT}

Non-cardiac critically ill patients with type II myocardial infarction (MI) have a high risk of mortality. There are no evidence-based interventions to mitigate this risk. We systematically reviewed the literature regarding the use of medications known to reduce mortality in patients with cardiac troponin (cTn) elevation due to type I MI ( $\beta$ blockers, statin, and aspirin) in studies of critically ill patients without Type I MI. All PubMed publications between 1976-2/19/16 were reviewed. Search terms included: $\beta$ blocker or aspirin or statin and intensive care unit (ICU) or critically ill or sepsis; 497 primary references were obtained. Inclusion criteria were as follows: (1) study population consisted of critically ill patients in the ICU with non-cardiovascular illnesses, (2) mortality end point, (3) severity of illness (or injury) was measured, and (4) the antiplatelet agent was primarily aspirin. Retrospective investigations, prospective observational studies, meta-analysis, systematic review, and randomized controlled trials were included; case reports were excluded. 25 primary references were obtained. The data were extracted and tabulated using data collection headings as follows: article title, first author/year/ reference number, study type/design, population studied, outcome and intervention, and study question addressed. Evidence was not graded as the majority of studies were non-randomized (low-tomoderate quality). 11 studies were found through bibliography reviews for a total of 36 references. In conclusion, $\beta$ blockers, statins, and aspirin may play a role in reducing mortality in non-cardiac critically ill patients. Benefit appears to be related to severity of illness, for which cTn may be a marker.

\section{INTRODUCTION}

Critically ill patients are defined as those having an acute impairment of one or more vital organ systems such that there is a high probability of imminent or life-threatening deterioration in condition. ${ }^{1}$ Despite significant resources provided for the treatment of the critically ill patients in the intensive care unit (ICU), the short-term mortality rate remains high. $^{23}$ Elevated serum cardiac troponin (cTn) is a common finding in this population, including those patients who do not initially present as cardiac emergencies, with $61 \%$ prevalence in patients with sepsis alone. ${ }^{4}$ Serum cTn is often measured to 'rule out' coronary arterial plaque rupture-or type I myocardial infarction $(\mathrm{MI})^{5}$-as a cause for observed hypotension, arrhythmias, or chest pain in the critically ill patients, since treatment with urgent revascularization of the obstructed artery would be recommended.

Obstructive coronary disease, however, is not often found in critically ill patients with cTn elevation. ${ }^{6}{ }^{7}$ Rather, cTn elevation in this setting is thought to be due to myocardial oxygen supply/demand mismatch, categorized as a type II MI. ${ }^{5}{ }^{8}$ In this context, it is not uncommon for patients to lack the signs and symptoms caused by plaque rupture which either may not be present or may be masked by sedation or the underlying illness (figure 1). Importantly, even in the absence of obstructive coronary disease, elevated cTn correlates with the severity of illness and is an independent predictor of death, ${ }^{4}{ }^{6}{ }^{9-25}$ although not all studies support this conclusion. ${ }^{26-30}$ To further complicate the situation, comorbidities such as hypotension, renal failure, or sepsis increase the risk of coronary intervention which, if performed, may cause more harm than benefit.

While the existing literature is robust regarding interventions and treatments known to reduce mortality for patients who present with type I MI, it lags behind in identifying interventions that reduce mortality in critically ill patients with type II MI and no prior coronary disease, despite the elevated mortality risk in this group. A common strategy has been to treat with $\beta$ blockers, statin, and aspirin while the patient recovers, assuming that obstructive coronary disease is present, and then pursue testing for coronary disease once the patient has stabilized (figure 1). Early mortality reduction with $\beta$ blockers, aspirin, or statin may provide a window of opportunity for subsequent evaluation and treatment of clinically significant coronary disease once the patient has stabilized from the underlying illness and the risks of intervention are mitigated.

This strategy has not been tested prospectively in critically ill patients with type II MI because there is little data in the literature to show benefit. To address this gap in knowledge, our group performed a retrospective study that included nearly 20,000 medical and surgical 


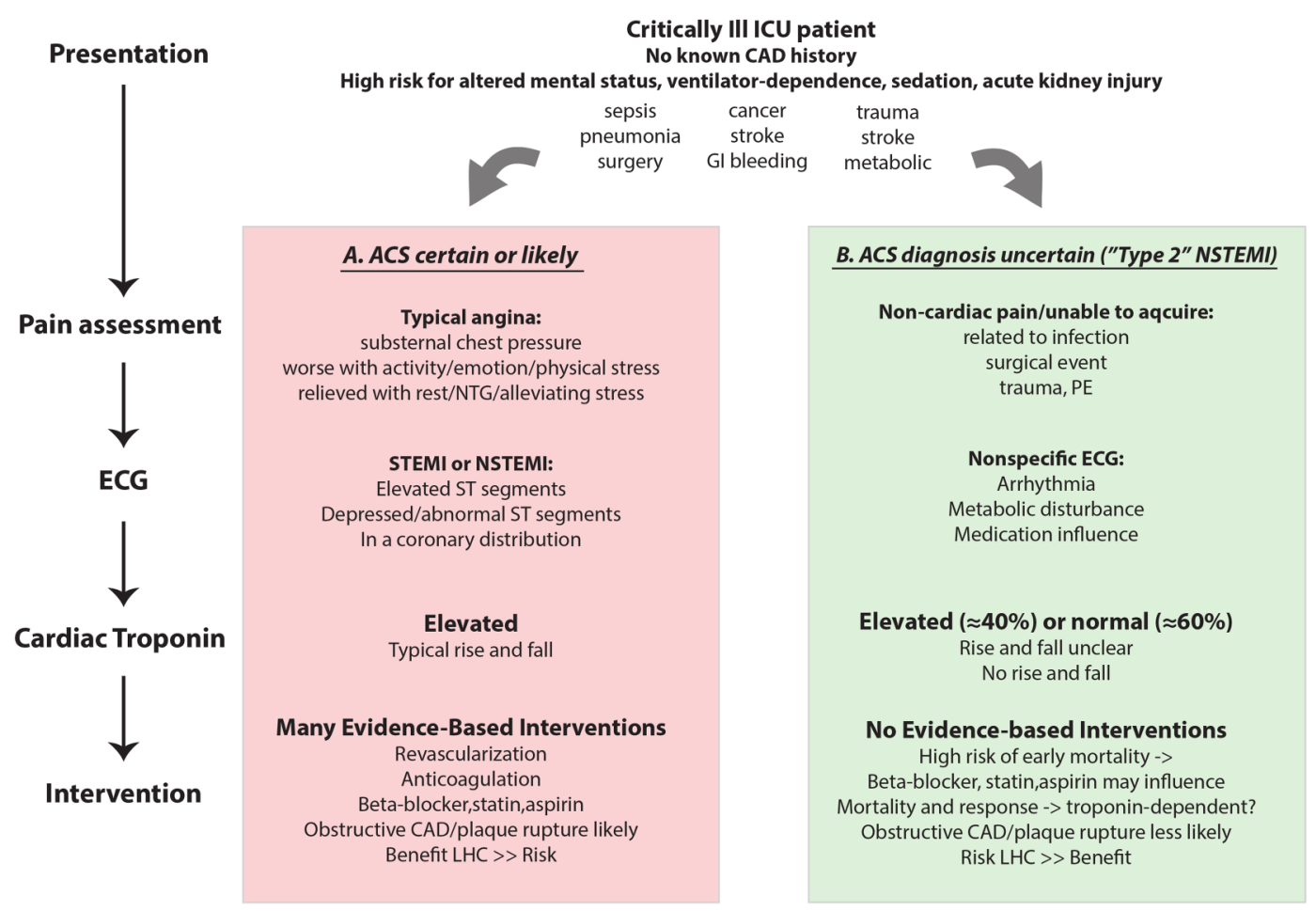

Figure 1 Assessment of the critically ill patient with troponin elevation. There is a lack of evidence-based interventions in high-risk critically ill patients with troponin elevations and no (or uncertain) acute coronary syndrome (ACS). ICU, intensive care unit; CAD, coronary artery disease.

patients who were admitted to Veterans Administration Medical Center ICUs from 2007 to $2008 .^{31}$ Our data revealed two important findings: (1) that cTn level correlated with the severity of illness, and (2) there was a 30-day mortality reduction in patients taking aspirin, $\beta$ blockers, and/or statins in a troponin-dependent fashion. Aspirin and/or $\beta$ blocker use was associated with a 30-day mortality reduction, but only if cTn levels were high ( $\beta$ blockers: $\mathrm{OR}=0.80 \quad(0.68,0.94), \mathrm{p}=0.0077$; aspirin: $\mathrm{OR}=0.81$ $(0.69,0.96), \mathrm{p}=0.0134)$. The 30 -day mortality was reduced in patients taking statins, but only if there was no or mild elevation of cTn $(\mathrm{OR}=0.66 \quad(0.53,0.82)$, $\mathrm{p}=0.0003$ ).

To the best of our knowledge, there is only one other report that demonstrated treatment benefit from medical intervention in a troponin-dependent fashion (see the 'Results' section). ${ }^{11}$ Given the correlation between serum cTn and severity of illness that has been replicated in many studies, we extended our search to examine the impact of $\beta$ blockers, aspirin, and statins in a critically ill population in which the severity of illness was also documented. Since $\beta$ blockers and aspirin were associated with reduced mortality in patients who had high cTn but not in those with normal or intermediate levels of $\mathrm{cTn}$, we hypothesized that $\beta$ blockers and aspirin would also be associated with reduced mortality in patients with a comparably high severity of illness. Likewise, since statins were associated with reduced mortality in critically ill patients with normal or intermediate levels of cTn, we hypothesized that we would see a mortality benefit in critically ill patients with lower severity of illness.

This is of great importance for several reasons. First, there are no known interventions that reduce mortality in critically ill patients with type II MI, a group at high risk of early death. Second, critically ill patients without cTn elevation may still benefit from statin use. All critically ill patients, therefore, may have mortality benefit from treatment with $\beta$ blocker, aspirin, or statins.

The goal of this systematic review is to determine whether there is a literature base to support additional investigation of clinical treatment pathways using $\beta$ blockers, statins, and/or aspirin in critically ill patients based on cTn or, in the absence of cTn levels, the severity of illness.

\section{METHODS}

\section{Data source and searches}

Literature searches were performed using PubMed February 19, 2016 and included the literature through 1976.

\section{Study selection}

Titles were scanned and abstracts reviewed using exclusion and inclusion criteria following PRISMA-P guidelines. ${ }^{32} 33$ Search terms included: $\beta$ blockers or aspirin or statin and ICU or critically ill or sepsis; 497 primary references were obtained. Inclusion criteria were as follows: (1) study population consisted of critically ill patients in the ICU with non-cardiovascular illnesses, (2) mortality end point, (3) severity of illness (or injury) was measured, and (4) the antiplatelet agent discussed was solely (7 studies) or largely (3 studies) aspirin. All languages were included. Retrospective investigations, prospective observational studies, meta-analysis, systematic review, and randomized controlled trials were included; case reports were excluded. Thirty-six primary references were obtained (figure 2). 


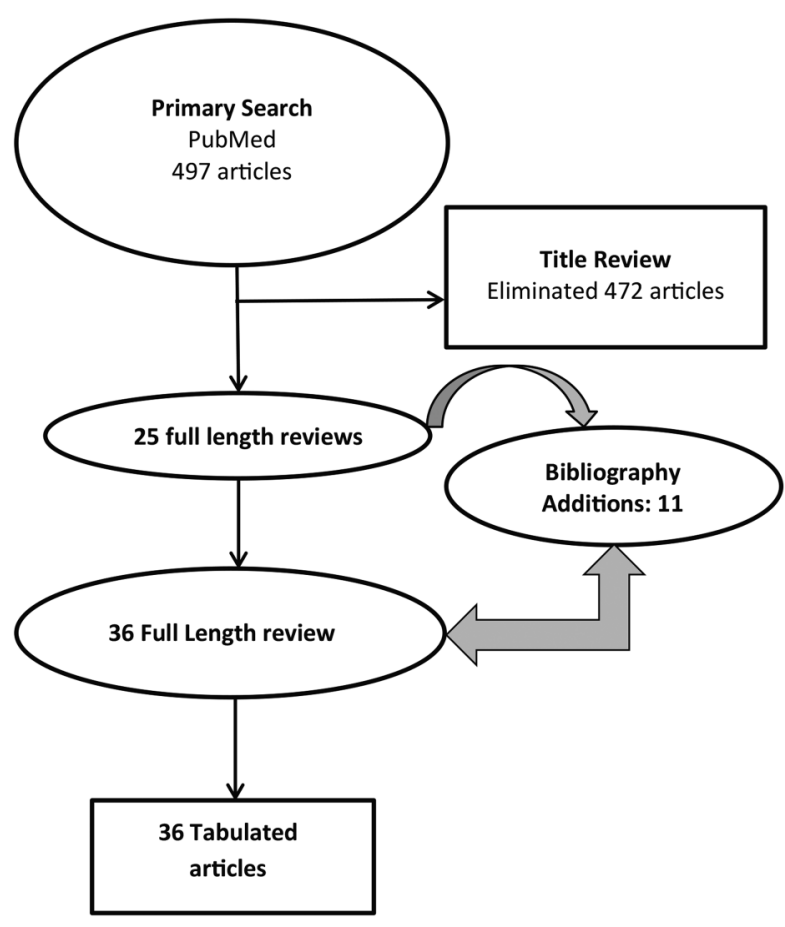

Figure 2 Methods: process of review. Literature reviewed.

\section{Data extraction and quality assessment}

The data were extracted and tabulated using data collection headings as follows: article title, first author and year, study type/design, population studied, outcome and intervention and study question addressed. Evidence was not graded as the majority of studies were non-randomized.

\section{Data synthesis and analysis}

The articles were obtained in full length and read by two independent reviewers (FGR and MBC). Eleven additional studies were found through bibliography reviews for a total of 36 references.

\section{RESULTS}

\section{Aspirin and antiplatelet agents}

Systematic review of the literature investigating the role of aspirin in critical illness suggests a potential benefit in patients who are more severely ill (see online supplementary table S1). Mechanisms of benefit may be related to coagulopathy, coagulopathy being associated with increased organ failure and death. ${ }^{34}$ Furthermore, organ failure contributes cumulatively to mortality. ${ }^{35}$ Therefore, interrupting the coagulation cascade has been examined as a method to limit organ failure in many investigations with different agents. Data regarding the antiplatelet agent aspirin are robust, but largely retrospective. None of the literature discussed here measured $\mathrm{cTn}$, however investigators measured and adjusted for the severity of illness or injury, in the case of critically injured trauma patients. The data suggest that aspirin may mitigate development of acute lung injury, ${ }^{36-40}$ acute respiratory distress syndrome, ${ }^{36} 41$ systemic inflammatory response syndrome, ${ }^{42}$ and mortality, ${ }^{37} 4243$ but these investigations need to be confirmed in appropriate prospective clinical trials.
The largest investigation of aspirin in the critically ill is a propensity-matched retrospective study of 7945 patients at risk for SIRS, APACHE II Score 17-18 (1445 patients in each group; this APACHE II Score predicts a mortality of 26-30\%). ${ }^{42}$ Aspirin users had a significantly reduced mortality compared to non-users (10.9\% aspirin users vs $17.2 \%$ non-users, HR $0.43, \mathrm{p}<0.001$ ), with benefit also in the sepsis-only group (27.4\% aspirin users vs $42.2 \%$ non-users, $95 \%$ CI $-18.8 \%$ to $-8.6 \%)$. An APACHE II score of $17-18$ is consistent with the report from Poe et $a l,{ }^{31}$ in which the patients who benefitted from aspirin use had a 30-day mortality of $30 \%$, as well as the highest cTn. This supports the proposal that selected critically ill patients with a high severity of illness score may benefit the most from aspirin use.

Another retrospective, propensity-matched investigation of 1149 critically ill patients with high severity of illness (APACHE II 25-29, predicted mortality $>50 \%$ ) found that prehospital use of aspirin was associated with a decreased risk of acute respiratory distress syndrome (ARDS) (multivariate-adjusted OR, 0.659 ; 95\% CI 0.46 to 0.94 ; $\mathrm{p}=0.023),{ }^{41}$ but only a trend towards decreased mortality in aspirin users (OR 0.697; 95\% CI 0.47 to 1.03, $\mathrm{p}=0.075)$. Although other medications were recorded, $\beta$ blockers as a class were not separately analyzed. Given the high risk of mortality in this patient population, it is possible that $\beta$ blocker administration confounded this result. Similarly, in a study of patients with severe sepsis, ${ }^{39}$ reduced incidence of ARDS/acute lung injury (ALI) was observed in the antiplatelet-treated group (OR $0.5,95 \% \mathrm{CI} 0.35$ to $0.71, \mathrm{p}<0.001)$, but no mortality benefit was demonstrated (OR 0.73 , 95\% CI 0.46 to 1.16 ), $\mathrm{p}=0.19) .{ }^{39}$ In this investigation, APACHE III scores (55-57) are associated with an anticipated mortality of $\sim 15-20 \%$, which would correlate to the 'intermediate cTn' group of Poe et al, a cohort that did not have an associated mortality benefit with aspirin. Once again, severity of illness may be predictive of outcome with aspirin use.

A substudy of the prospectively enrolled Glue Grant found benefit from aspirin. ${ }^{37}$ High-risk trauma patients who received transfusions (a risk factor for postinjury pulmonary dysfunction) were enrolled. Of 839 patients, 128 patients were on antiplatelet agents; $66 \%$ aspirin alone, $20 \%$ 'other' antiplatelet agents, and 14\% received aspirin and another antiplatelet agent. Despite the fact that patients on antiplatelet agents were older, had more comorbid illnesses and were more severely injured, patients on antiplatelet agents had significantly less multi-organ failure and lung dysfunction with a non-significant trend towards decreased mortality.

A retrospective study of 979 septic patients (mean APACHE II score 22-23, p=0.16) showed mortality benefit from aspirin. ${ }^{44}$ Logistic regression analysis determined that being on aspirin or a non-steroidal anti-inflammatory agent (NSAID; ibuprofen, diclofenac, or indomethacin) was associated with decreased mortality (aspirin (ASA) OR 0.57, $95 \%$ CI 0.39 to 0.83 , NSAID OR 0.5 , CI 0.26 to 0.94 ); however, being on both agents eliminated the benefit of either agent (ASA and NSAID OR 1.12, CI 0.55 to 2.25), indicating that NSAID use must be considered in clinical trials.

Even patients with severe gastrointestinal bleeding may have a mortality benefit with aspirin. A total of 717 patients admitted for non-variceal upper gastrointestinal 
bleeding from 1993 to 2010 were studied. ${ }^{45}$ The primary outcome was in-hospital mortality. Despite the fact that patients on ASA only were older and had more comorbidities, multivariate analysis showed that being on ASA only was an independent predictor of reduced in-hospital mortality (OR $0.26,95 \%$ CI 0.13 to $0.53, \mathrm{p}=0.0002$ ).

A propensity-matched study of 3855 patients investigating aspirin use in patients at risk for acute lung injury found no benefit from aspirin (OR 0.67; 95\% CI 0.44 to $1.01, \mathrm{p}=0.055)$; however, the APACHE II score was relatively low, between 9 and 12 (approximate mortality risk $15 \%) .{ }^{38}$ The reduced benefit may have been related to the reduced risk in this cohort. A retrospective study of mixed admissions to medical and surgical ICUs supports this assertion. ${ }^{46}$ Patients with an APACHE II score of 21 or greater had a significant benefit from antiplatelet pretreatment as compared to patients with lower APACHE II scores. Severity of illness, or cTn levels if they are indeed a biomarker of severity of illness, may need to be considered when prescribing aspirin. ${ }^{31} 37$

One nested cohort trial of 763 ICU patients (20\% receiving aspirin) combined results from two RCTs and showed potential harm from aspirin therapy. ${ }^{47}$ The authors did not provide a mechanism for this difference as compared with considerable literature showing benefit, rather they highlighted the need for prospective, randomized investigation.

In summary, systematic review of a series of large, retrospective studies of critically ill patients suggests that aspirin provides clinical benefit, particularly when the severity of illness is high, although this finding is not uniform. This supports the need for prospective, randomized, controlled investigations of aspirin in the critically ill, with careful evaluation of the possible role of severity of illness and troponin positivity.

\section{Statins}

Statins hold promise in the treatment of sepsis, primarily via immunomodulatory and anti-inflammatory mechanisms. ${ }^{48}$ Systematic review of the literature describing statin use in the critically ill revealed a confusing story, but one that may be clarified by addressing severity of illness. Observational cohort investigations, retrospective and prospective, have demonstrated a potential benefit of statins in patients with sepsis, ${ }^{49}$ but the bulk of data from prospective, randomized, blinded, placebo-controlled trials suggests otherwise. ${ }^{50-53}$ The remainder of this discussion will focus on results from prospective randomized-controlled trials and their meta-analyses.

The conclusion from recent systematic reviews and meta-analyses is that there is insufficient evidence to support the use of statins to derive a mortality benefit in patients with severe sepsis. ${ }^{54-57}$ A total of 1894 patients were involved in randomized, blinded, and controlled clinical trials of statin therapy in sepsis. Primary outcome was 28-day mortality, ${ }^{50}$ in-hospital mortality, ${ }^{51}$ number of ventilator-free days for a maximum of 28 days, ${ }^{52}$ hemodynamic parameters, ${ }^{58}$ and IL- 6 levels. ${ }^{53}$ None of the trials showed mortality benefit except in patients who were prior users of statin (Kruger; $n=77$ patients, OR 0.17 (0.03 to $0.85), p=0.03) .{ }^{53}$ Patients in these trials had a high severity of illness, with approximate APACHE II scores ranging from 19 to 25 and estimated mortality rates between 30\% and 53\%. It was concluded that severely septic patients do not benefit from statin use, although it is not clear if prior use impacts mortality. ${ }^{53}$

Severity of illness may play an important role in determining whether a patient will benefit from statins, as suggested by a large retrospective cohort analysis ${ }^{31}$ and one randomized, controlled, double blind study. ${ }^{31} 59$ Among 16,208 critically ill patients with a severity of illness comparable to APACHE II score of 14 or less, logistic regression analysis showed that patients taking statins had an associated 30-day mortality benefit (OR 0.66; 95\% CI 0.53 to $0.82, \mathrm{p}=0.0003) .{ }^{31}$ Patients with a higher severity of illness (equivalent to APACHE II score >19) did not have an associated mortality benefit with statin use (OR 0.99 (95\% CI 0.82 to 1.19$), \mathrm{p}=0.91)$. This retrospective study could not adjust for prior statin use, however. It is possible that the entire effect observed may be related to prior statin use rather than severity of illness.

A randomized, placebo-controlled, double blind investigation of statin use in septic patients on the ward showed that $40 \mathrm{mg}$ of atorvastatin in statin-naïve patients prevented conversion of sepsis to severe sepsis (4\% vs $24 \%$, $\mathrm{p}=0.007) .{ }^{59}$ The mean APACHE II score for this group correlated to an anticipated mortality rate of $15 \%$.

This systematic review of prospective, randomized, controlled studies of statins in the critically ill suggests that statin use may benefit patients who are less severely ill on admission, supporting an immunomodulatory effect beneficial at early stages of illness. The impact of prior use of statins also needs to be clarified. It is possible that most benefit derives from prior use, but at least one randomized trial of septic ward patients suggests that statin-naïve patients may benefit, as well.

\section{B blockade}

Systematic review of the literature reveals that $\beta$ blockade may reduce mortality in critically ill patients who are more severely injured or ill, or who have higher cTn levels on admission. Only one prospective, randomized, placebocontrolled clinical investigation of the impact of $\beta$ blockade on mortality in the critically ill has been published. ${ }^{60} \mathrm{~A}$ total of 154 patients who presented with hemodynamically unstable septic shock requiring norepinephrine were investigated. After careful resuscitation, the patients randomized to treatment were given intravenous esmolol. The primary outcome was a reduction in heart rate to $80-94 \mathrm{bpm}$ and maintained for 96 hours, which was achieved; the mean reduction in heart rate in the esmolol group was $18 \mathrm{bpm}$ $(\mathrm{p}<0.001)$. Surprisingly, at 28 days, the esmolol group had a mortality rate of $49.4 \%$, whereas the control group was $80.5 \%(\mathrm{p}<0.001)$. The authors pointed out that historically, patients with the highest heart rates were most at risk of death ${ }^{61-65}$ and hypothesized that perhaps the highest risk patients would benefit most from $\beta$ blockade.

Several prospective observational studies suggest $\beta$ blockers may reduce mortality in patients with traumatic brain injury (TBI). A non-randomized, prospective, observational study of 440 TBI patients was performed in which propranolol was given within the first 24 hours of ICU admission at the discretion of the trauma or neurosurgery attending doctors. ${ }^{66}$ Propranolol-a non-cardioselective $\beta$ blocker-was chosen based on a retrospective 
investigation of 1755 TBI patients which showed that there was no in-hospital mortality benefit from $\beta$ blockade in general (427 patients, multivariable analysis OR $0.85,95 \%$ CI 0.536 to 1.348$)$. However 78 propranolol users $(18 \%)$ alone had significant mortality benefit (OR 0.199, 95\% CI 0.043 to 0.92 ) despite being more severely injured. ${ }^{67}$ In the prospective observational investigation, propranolol use was independently associated with reduced early mortality (OR $0.25 \quad(0.08,0.74, \quad \mathrm{p}=0.012)){ }^{66}$ A second prospective observational study of propranolol use with 38 moderate-to-severe TBI patients (28 propranolol group, 10 control patients) supported its safety, but did not show a mortality benefit $(10 \%$ vs $10.7 \%, p=0.9) .{ }^{68}$ This may reflect the fact that $80 \%$ of patients in the non-propranolol group had been given other forms of $\beta$ blockade during their admission. Clearly, additional high-quality prospective investigations are necessary to show benefit of $\beta$ blockade in TBI patients as well as investigating the importance of cardioselectivity; however, this early work indicates that there is little harm. Support for lack of harm and possible benefit in TBI patients also comes from considerable retrospective work, ${ }^{69-72}$ all of which suggests that $\beta$ blockade has a protective effect despite higher risk injuries.

$\beta$ blockade also appears to show mortality benefit in critically ill non-TBI trauma patients. ${ }^{11} 73{ }^{74}$ Martin et al ${ }^{11}$ investigated the role of cTn elevation in 1081 trauma patients and asked whether $\beta$ blockers and aspirin impacted outcomes. Consistent with the majority of similar investigations, the authors found increased mortality in trauma patients who also had elevated cTn (29\% of patients with elevated cTn, mortality $16 \%$ in cTn negative vs $44 \%$ in $\mathrm{cTn}$ positive groups, OR 3.0, $\mathrm{p}<0.01)$. APACHE II score was an independent predictor of mortality $(p=0.001)$. Seven per cent of patients were administered $\beta$ blockers, primarily to patients with elevated cTn $(11 \%$ vs $6 \%$, $p=0.01)$. $\beta$ blocker use was associated with a $50 \%$ reduction in mortality, but only in the group with elevated cTn (cTn elevated: $38 \%$ vs $16 \%, \mathrm{p}<0.01$, cTn not elevated: $14.3 \%$ vs $16 \%, \mathrm{p}=0.77)$. Adjusting for multiple factors still resulted in a strong mortality benefit (OR $0.50 ; 95 \%$ CI 0.24 to $1.02, p=0.057)$. This is consistent with the work of Poe et al, ${ }^{31}$ which showed similar benefit across the critical illness spectrum in patients with high cTn as compared to patients with normal or mildly elevated levels.

Considerable retrospective work shows an association with $\beta$ blockade and reduced mortality in the nontraumatic critically ill. A large propensity-adjusted cohort study used Denmark's prospective, population-based medical database. ${ }^{75}$ Thirty-day mortality was measured in 8087 ICU patients taking $\beta$ blockers prior to admission as compared to non-users. Similar to other studies, despite the fact that $\beta$ blocker users were older and had more comorbid diseases than non-users, there was significant association of mortality benefit with cardioselective agents (OR $0.70 ; 95 \%$ CI 0.58 to 0.83 ). A retrospective cohort study comparing heart-rate lowering agents in patients admitted to the ICU in acute respiratory failure showed no mortality benefit among 188 chronic obstructive pulmonary syndrome (COPD) patients. $^{76}$ This population, however, had a severity of illness (APACHE II 19-20) that may have also benefited from aspirin use, if the work of Poe $e t a l^{31}$ is accurate. Mortality benefit, therefore, may have been masked by aspirin use which was not addressed in this study.

Additional retrospective cohort studies of non-critically ill patients with COPD support the safety of $\beta$ blockade. ${ }^{77-79}$ Three investigations showed early mortality benefit with $\beta$ blocker use in non-critically ill patients with $\mathrm{COPD}^{77} 78$ or respiratory arrest. ${ }^{79}$ The majority of benefit was seen in patients with cardiovascular disease in a study of noncritically ill COPD patients ${ }^{78}$ and respiratory arrest patients. ${ }^{79}$ When patients with cardiovascular disease are excluded, a mortality benefit remained, adjusted HR 0.68 (95\% CI 0.46 to 1.02$){ }^{77}$

\section{SUMMARY AND CONCLUSIONS}

Reasonable conclusions that can be cautiously extracted from this systematic review are as follows:

1. Cardiac troponin levels may be a biomarker for severity of illness and multiorgan failure, thus providing a potential method to rapidly identify those patients at greatest risk of death for whom $\beta$ blockers and aspirin may have the greatest benefit.

2. Aspirin appears to reduce acute lung injury, occurrence of ARDS, and may also impact mortality in critically ill patients. The most severely ill may obtain the most benefit. This needs to be tested in prospective, randomized, blinded, and controlled investigations.

3. Prospective, randomized, and controlled investigations show that statins appear to have no benefit in reducing mortality or illness in severely septic patients. Some data suggest overall safety in septic patients. This impacts patients who have other indications for statins, such as cardiovascular disease. Close surveillance of liver and muscle biomarkers is recommended due to the potential for liver and muscle dysfunction in the severely ill.

4. Conclusions cannot yet be drawn regarding the benefits of statins in less severely ill patients. In this population, statins may reduce the severity of illness and may have a mortality benefit, but this also needs to be confirmed with appropriate prospective interventional trials.

5. In the one prospective, randomized, controlled study of $\beta$ blockers in adequately resuscitated septic patients, $\beta$ blockers appear to have significant mortality benefit, although there has not yet been such an investigation designed with a mortality end point. It is possible that cardioselectivity may be important, although esmolol (cardioselective) and propranolol (non-selective) have shown mortality reduction in small studies of patients with sepsis and TBI, respectively. Side-by-side prospective studies of both classes of $\beta$ blocker would be necessary to address this question.

6. All medications must be recorded and considered in future studies of critical illness.

This systematic review cumulatively suggests that statin therapy may provide immunomodulatory benefits at early stages of illness before multiorgan failure develops, whereas aspirin and $\beta$ blockers may reduce mortality in the sickest of patients for whom platelet aggregation, microvascular obstruction, and organ dysfunction may occur.

A treatment pathway that needs to be tested prospectively is summarized in figure 3. Potentially, all non-acute coronary syndrome (ACS) admissions to the ICU would 


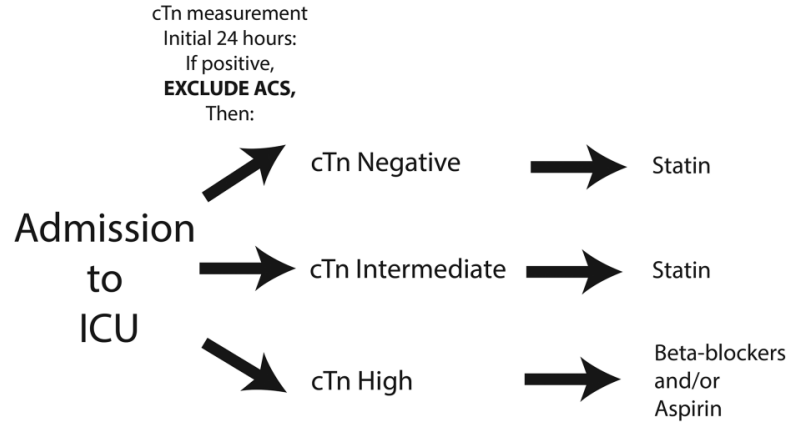

Figure 3 Measurement of cTn in critically ill patients may help guide potentially life-saving therapy. Consideration for assessment of patients with type II myocardial infarction. Modified from Poe et al. ${ }^{31}$

trigger a baseline cTn measurement that could guide appropriate medical management. After excluding the possibility of ACS and assessing patient-specific risks and benefits, it is possible that the addition of $\beta$ blockers, statins, and/or aspirin would reduce mortality in critically ill patients depending on the severity of illness, a surrogate marker being the admission cTn level. Prospective, observational studies addressing these questions must be developed.

Contributors $\mathrm{FGR}, \mathrm{MBC}, \mathrm{HJ}$ and RHV-P have all contributed to the conception or design of the work, in revising this work critically for important intellectual content, have all contributed to the final version and have all agreed to be accountable for all aspects of the work

Disclaimer This material is the result of work supported with resources and the use of facilities at the Cincinnati VAMC.

Competing interests None declared.

Provenance and peer review Not commissioned; externally peer reviewed.

Open Access This is an Open Access article distributed in accordance with the Creative Commons Attribution Non Commercial (CC BY-NC 4.0) license, which permits others to distribute, remix, adapt, build upon this work noncommercially, and license their derivative works on different terms, provided the original work is properly cited and the use is non-commercial. See: http:/l creativecommons.org/licenses/by-nc/4.0/

\section{REFERENCES}

1 Nates IL, Nunnally M, Kleinpell R, et al. ICU Admission, Discharge, and Triage Guidelines: a framework to enhance clinical operations, development of institutional policies, and further research. Crit Care Med 2016;44:1553-602.

2 Angus DC, Linde-Zwirble WT, Lidicker J, et al. Epidemiology of severe sepsis in the United States: analysis of incidence, outcome, and associated costs of care. Crit Care Med 2001;29:1303-10.

3 Dellinger RP, Levy MM, Rhodes A, et al. Surviving sepsis campaign: international guidelines for management of severe sepsis and septic shock, 2012. Intensive Care Med 2013;39:165-228.

4 Bessière $F$, Khenifer $S$, Dubourg J, et al. Prognostic value of troponins in sepsis: a meta-analysis. Intensive Care Med 2013;39:1181-9.

5 Thygesen $\mathrm{K}$, Alpert JS, Jaffe AS, et al. Third universal definition of myocardial infarction. J Am Coll Cardiol 2012;60:1581-98.

6 Ammann P, Maggiorini M, Bertel O, et al. Troponin as a risk factor for mortality in critically ill patients without acute coronary syndromes. J Am Coll Cardiol 2003:41:2004-9.

7 Baron T, Hambraeus K, Sundström J, et al. TOTAL-AMI Study Group. Type 2 myocardial infarction in clinical practice. Heart 2015;101:101-6.

8 Alpert JS, Thygesen KA, White HD, et al. Diagnostic and therapeutic implications of type 2 myocardial infarction: review and commentary. Am J Med 2014;127:105-8.

9 Stein R, Gupta B, Agarwal S, et al. Prognostic implications of normal $(<0.10$ $\mathrm{ng} / \mathrm{ml})$ and borderline $(0.10$ to $1.49 \mathrm{ng} / \mathrm{ml})$ troponin elevation levels in critically ill patients without acute coronary syndrome. Am J Cardiol 2008;102:509-12.

10 Salim A, Hadjizacharia P, Brown C, et al. Significance of troponin elevation after severe traumatic brain injury. J Trauma 2008;64:46-52.

11 Martin M, Mullenix $\mathrm{P}$, Rhee $\mathrm{P}$, et al. Troponin increases in the critically injured patient: mechanical trauma or physiologic stress? I Trauma 2005;59:1086-91.

12 Lim W, Qushmaq I, Devereaux PJ, et al. Elevated cardiac troponin measurements in critically ill patients. Arch Intern Med 2006;166:2446-54.

13 Reynolds T, Cecconi M, Collinson P, et al. Raised serum cardiac troponin I concentrations predict hospital mortality in intensive care unit patients. Br J Anaesth 2012;109:219-24.

14 Baillard C, Boussarsar M, Fosse JP, et al. Cardiac troponin I in patients with severe exacerbation of chronic obstructive pulmonary disease. Intensive Care Med 2003:29:584-9.

15 Vasile VC, Chai HS, Abdeldayem D, et al. Elevated cardiac troponin T levels in critically ill patients with sepsis. Am J Med 2013;126:1114-21.

16 Wu TT, Yuan A, Chen CY, et al. Cardiac troponin I levels are a risk factor for mortality and multiple organ failure in noncardiac critically ill patients and have an additive effect to the APACHE II score in outcome prediction. Shock 2004;22:95-101.

17 Quenot JP, Le Teuff TG, Quantin C, et al. Myocardial injury in critically ill patients: relation to increased cardiac troponin I and hospital mortality. Chest 2005:128:2758-64.

18 John J, Woodward DB, Wang Y, et al. Troponin-I as a prognosticator of mortality in severe sepsis patients. J Crit Care 2010;25:270-5.

19 Wright RS, Williams BA, Cramner $\mathrm{H}$, et al. Elevations of cardiac troponin I are associated with increased short-term mortality in noncardiac critically ill emergency department patients. Am J Cardiol 2002;90:634-6.

20 Giannitsis E, Müller-Bardorff M, Kurowski V, et al. Independent prognostic value of cardiac troponin $\mathrm{T}$ in patients with confirmed pulmonary embolism. Circulation 2000;102:211-17.

21 Jensen JK, Kristensen SR, Bak S, et al. Frequency and significance of troponin T elevation in acute ischemic stroke. Am J Cardiol 2007:99:108-12.

22 Babuin L, Vasile VC, Rio Perez JA, et al. Elevated cardiac troponin is an independent risk factor for short- and long-term mortality in medical intensive care unit patients. Crit Care Med 2008;36:759-65.

23 Vasile VC, Chai HS, Khambatta S, et al. Significance of elevated cardiac troponin T levels in critically ill patients with acute respiratory disease. Am J Med 2010;123:1049-58.

24 Hassan B, Morsy S, Siam A, et al. Myocardial injury in critically ill children: a case control study. ISRN Cardiol 2014;2014:919150.

25 Lim W, Cook DJ, Griffith LE, et al. Elevated cardiac troponin levels in critically ill patients: prevalence, incidence, and outcomes. Am J Crit Care 2006;15:280-8.

26 Brivet FG, Jacobs FM, Colin P, et al. Cardiac troponin level is not an independent predictor of mortality in septic patients requiring medical intensive care unit admission. Crit Care 2006:10:404.

27 King DA, Codish S, Novack V, et al. The role of cardiac troponin I as a prognosticator in critically ill medical patients: a prospective observational cohort study. Crit Care 2005;9:R390-5.

28 Kalla C, Raveh D, Algur N, et al. Incidence and significance of a positive troponin test in bacteremic patients without acute coronary syndrome. Am J Med 2008;121:909-15.

29 Tiruvoipati R, Sultana N, Lewis D. Cardiac troponin I does not independently predict mortality in critically ill patients with severe sepsis. Emerg Med Australas 2012;24:151-8

30 Oliveira NS, Silva VR, Castelo JS, et al. Serum level of cardiac troponin I in pediatric patients with sepsis or septic shock. Pediatr Crit Care Med 2008:9:414-17.

31 Poe S, Vandivier-Pletsch RH, Clay M, et al. Cardiac troponin measurement in the critically ill: potential for guiding clinical management. I Investig Med 2015;63:905-15

32 Shamseer L, Moher D, Clarke M, et al. PRISMA-P Group. Preferred reporting items for systematic review and meta-analysis protocols (PRISMA-P) 2015: elaboration and explanation. BMJ 2015;349:g7647.

33 Moher D, Shamseer L, Clarke M, et al. Preferred reporting items for systematic review and meta-analysis protocols (PRISMA-P) 2015 statement. Syst Rev 2015:4:1.

34 Dhainaut JF, Shorr AF, Macias WL, et al. Dynamic evolution of coagulopathy in the first day of severe sepsis: relationship with mortality and organ failure. Crit Care Med 2005;33:341-8.

35 Martin GS, Mannino DM, Eaton S, et al. The epidemiology of sepsis in the United States from 1979 through 2000. N Engl J Med 2003;348: 1546-54. 
36 O'Neal HR Jr, Koyama T, Koehler EA, et al. Prehospital statin and aspirin use and the prevalence of severe sepsis and acute lung injury/acute respiratory distress syndrome. Crit Care Med 2011;39:1343-50.

37 Harr JN, Moore EE, Johnson J, et al. Antiplatelet therapy is associated with decreased transfusion-associated risk of lung dysfunction, multiple organ failure, and mortality in trauma patients. Crit Care Med 2013;41: 399-404.

38 Kor DJ, Erlich J, Gong MN, et al. Association of prehospitalization aspirin therapy and acute lung injury: results of a multicenter international observational study of at-risk patients. Crit Care Med 2011;39:2393-400.

39 Valerio-Rojas JC, Jaffer IJ, Kor DJ, et al. Outcomes of severe sepsis and septic shock patients on chronic antiplatelet treatment: a historical cohort study. Crit Care Res Pract 2013;2013:782573.

40 Erlich JM, Talmor DS, Cartin-Ceba R, et al. Prehospitalization antiplatelet therapy is associated with a reduced incidence of acute lung injury: a population-based cohort study. Chest 2011;139:289-95.

41 Chen W, Janz DR, Bastarache JA, et al. Prehospital aspirin use is associated with reduced risk of acute respiratory distress syndrome in critically ill patients: a propensity-adjusted analysis. Crit Care Med 2015;43:801-7.

42 Eisen DP, Reid D, McBryde ES. Acetyl salicylic acid usage and mortality in critically ill patients with the systemic inflammatory response syndrome and sepsis. Crit Care Med 2012;40:1761-7.

43 Otto GP, Sossdorf M, Boettel J, et al. Effects of low-dose acetylsalicylic acid and atherosclerotic vascular diseases on the outcome in patients with severe sepsis or septic shock. Platelets 2013;24:480-5.

44 Sossdorf M, Otto GP, Boettel J, et al. Benefit of low-dose aspirin and nonsteroidal anti-inflammatory drugs in septic patients. Crit Care 2013;17:402.

45 Wehbeh A, Tamim HM, Abu Daya $\mathrm{H}$, et al. Aspirin has a protective effect against adverse outcomes in patients with nonvariceal upper gastrointestinal bleeding. Dig Dis Sci 2015;60:2077-87.

46 Winning J, Neumann J, Kohl M, et al. Antiplatelet drugs and outcome in mixed admissions to an intensive care unit. Crit Care Med 2010;38:32-7.

47 Al Harbi SA, Tamim HM, Al-Dorzi HM, et al. Association between aspirin therapy and the outcome in critically ill patients: a nested cohort study. BMC Pharmacol Toxicol 2016;17:5.

48 Terblanche M, Almog Y, Rosenson RS, et al. Statins and sepsis: multiple modifications at multiple levels. Lancet Infect Dis 2007;7:358-68.

49 Wan YD, Sun TW, Kan QC, et al. Effect of statin therapy on mortality from infection and sepsis: a meta-analysis of randomized and observational studies. Crit Care 2014;18:R71.

50 Papazian L, Roch A, Charles PE, et al. Effect of statin therapy on mortality in patients with ventilator-associated pneumonia: a randomized clinical trial. JAMA 2013;310:1692-700.

51 Truwit JD, Bernard GR, Steingrub J, et al. Rosuvastatin for sepsis-associated acute respiratory distress syndrome. N Engl J Med 2014;370:2191-200.

52 McAuley DF, Laffey JG, O'Kane CM, et al. Simvastatin in the acute respiratory distress syndrome. N Engl J Med 2014;371:1695-703.

53 Kruger $\mathrm{P}$, Bailey $\mathrm{M}$, Bellomo $\mathrm{R}$, et al. A multicenter randomized trial of atorvastatin therapy in intensive care patients with severe sepsis. Am J Respir Crit Care Med 2013;187:743-50.

54 Tralhão AF, Cés de Souza-Dantas V, Salluh Jl, et al. Impact of statins in outcomes of septic patients: a systematic review. Postgrad Med 2014;126:45-58.

55 Thomas G, Hraiech S, Loundou A, et al. Statin therapy in critically-ill patients with severe sepsis: a review and meta-analysis of randomized clinical trials. Minerva Anestesiol 2015;81:921-30.

56 Pasin L, Landoni G, Castro ML, et al. The effect of statins on mortality in septic patients: a meta-analysis of randomized controlled trials. PLoS One 2013;8:e82775

57 Deshpande A, Pasupuleti V, Rothberg MB. Statin therapy and mortality from sepsis: a meta-analysis of randomized trials. Am J Med 2015;128:410-17.
58 El Gendy HA, Elsharnouby NM. Safety and vasopressor effect of rosuvastatin in septic patients. Eg J Anaesth 2014;30:311-17.

59 Patel JM, Snaith C, Thickett DR, et al. Randomized double-blind placebo-controlled trial of $40 \mathrm{mg} /$ day of atorvastatin in reducing the severity of sepsis in ward patients (ASEPSIS Trial). Crit Care 2012;16:R231.

60 Morelli A, Ertmer C, Westphal M, et al. Effect of heart rate control with esmolol on hemodynamic and clinical outcomes in patients with septic shock: a randomized clinical trial. JAMA 2013;310:1683-91.

61 Schmittinger CA, Dünser MW, Haller M, et al. Combined milrinone and enteral metoprolol therapy in patients with septic myocardial depression. Crit Care 2008;12:R99.

62 Sander $\mathrm{O}$, Welters ID, Foëx $\mathrm{P}$, et al. Impact of prolonged elevated heart rate on incidence of major cardiac events in critically ill patients with a high risk of cardiac complications. Crit Care Med 2005;33:81-8; discussion 241-2.

63 Parker MM, Shelhamer JH, Natanson C, et al. Serial cardiovascular variables in survivors and nonsurvivors of human septic shock: heart rate as an early predictor of prognosis. Crit Care Med 1987;15:923-9.

64 Luckner G, Dunser MW, Jochberger S, et al. Arginine vasopressin in 316 patients with advanced vasodilatory shock. Crit Care Med 2005;33:2659-66.

65 Benbenishty J, Weissman C, Sprung CL, et al. Characteristics of patients receiving vasopressors. Heart Lung 2011;40:247-52.

66 Ko A, Harada MY, Barmparas G, et al. Early propranolol after traumatic brain injury is associated with lower mortality. I Trauma Acute Care Surg 2016;80:637-42. doi: 10.1097/TA.0000000000000959

67 Schroeppel TJ, Sharpe JP, Magnotti LJ, et al. Traumatic brain injury and beta-blockers: not all drugs are created equal. J Trauma Acute Care Surg 2014;76:504-9.

68 Murry JS, Hoang DM, Barmparas G, et al. Prospective evaluation of early propranolol after traumatic brain injury. J Surg Res 2016;200:221-6.

69 Schroeppel TJ, Fischer PE, Zarzaur BL, et al. Beta-adrenergic blockade and traumatic brain injury: protective? J Trauma 2010;69:776-82.

70 Riordan WP Jr, Cotton BA, Norris PR, et al. Beta-blocker exposure in patients with severe traumatic brain injury (TBI) and cardiac uncoupling. J Trauma 2007:63:503-10

71 Inaba K, Teixeira PG, David JS, et al. Beta-blockers in isolated blunt head injury. J Am Coll Surg 2008;206:432-8.

72 Cotton BA, Snodgrass KB, Fleming SB, et al. Beta-blocker exposure is associated with improved survival after severe traumatic brain injury. J Trauma 2007;62:26-33.

73 Arbabi S, Campion EM, Hemmila MR, et al. Beta-blocker use is associated with improved outcomes in adult trauma patients. J Trauma 2007;62: 56-61.

74 Bukur $\mathrm{M}$, Lustenberger $\mathrm{T}$, Cotton $\mathrm{B}$, et al. Beta-blocker exposure in the absence of significant head injuries is associated with reduced mortality in critically ill patients. Am J Surg 2012;204:697-703.

75 Christensen S, Johansen MB, Tønnesen E, et al. Preadmission beta-blocker use and 30-day mortality among patients in intensive care: a cohort study. Crit Care 2011;15:R87.

76 Kargin F, Takir HB, Salturk C, et al. The safety of beta-blocker use in chronic obstructive pulmonary disease patients with respiratory failure in the intensive care unit. Multidiscip Respir Med 2014;9:8.

77 Rutten FH, Zuithoff NP, Hak E, et al. Beta-blockers may reduce mortality and risk of exacerbations in patients with chronic obstructive pulmonary disease. Arch Intern Med 2010;170:880-7.

$78 \mathrm{Au}$ DH, Bryson CL, Fan VS, et al. Beta-blockers as single-agent therapy for hypertension and the risk of mortality among patients with chronic obstructive pulmonary disease. Am J Med 2004;117:925-31.

79 Noveanu M, Breidthardt T, Reichlin T, et al. Effect of oral beta-blocker on short and long-term mortality in patients with acute respiratory failure: results from the BASEL-II-ICU study. Crit Care 2010;14:R198. 